\title{
Towards a durable management of genetic resistances to Leptosphaeria maculans
}

Oléagineux, Corps Gras, Lipides. Volume 10, Numéro 3, 208-11, Mai 2003, Colza : enjeux et nouvelles synergies de la recherche, RECHERCHE

Auteur(s) : Xavier PINOCHET ${ }^{1}$, Emmanuelle MESTRIES ${ }^{1}$, Annette PENAUD ${ }^{1}$, Régine DELOURME ${ }^{2}$, Anne-Marie CHEVRE ${ }^{2}$, Michel RENARD ${ }^{2}$, Hortense BRUN ${ }^{3}$, Lydia BOUSSET ${ }^{3}$ Marie-Hélène BALESDENT ${ }^{4}$, Thierry ROUXEL ${ }^{4}$, Jean-Noël AUBERTOT ${ }^{5}$

${ }^{1}$ CETIOM Grignon, Centre de Grignon, BP 4, 78850 Thiverval-Grignon

${ }^{2}$ URM INRA ENSAR amélioration des plantes et biotechnologies végétales, Rennes

${ }^{3}$ UMR INRA ENSAR Bio3P Rennes

${ }^{4}$ INRA pathologie végétale Versailles

${ }^{5}$ INRA-INA-PG Agronomie Grignon

Summary : Blackeg is the major disease of winter oilseed rape in France. For an efficient control of the disease several tools could be combined, but mainly plant breeding has been used to increase winter oilseed rape resistance to blackleg. A useful step was reached in the nineties, using a specific resistance gene (RIm1). After being widely used this resistance was broken down by an increase of the virulent sub-populations of the fungus. Such a situation worried the different actors and raised the question of the promotion of a durable management of genetic resistances to Leptosphaeria. After a synthetic presentation of the state of the art in the fields of genetics of resistance, of Leptosphaeria populations and on agronomic practices able to control the pathogen, the promotion of a durable management of resistances is discussed. This target needs to precise the strategy, to improve methodologies to characterise genotypes, to promote proper agronomic practices, to follow Leptosphaeria population behaviour and to motivate economic actors.

Keywords : Oilseed rape, Leptosphaeria, blackleg, genetic resistances, durable management.

\section{ARTICLE}

Blackleg is the major disease of winter oilseed rape in France caused by the fungus Leptosphaeria maculans. Its impact on yield decrease was estimated between 5 and $20 \%$ of the French national oilseed rape production. For an efficient control of the disease several tools could be combined. Except in specific conditions, fungicide applications are often of poor interest due to a lack of persistency compared to the duration of fungal ascospore dissemination. Successively reduced applications are currently being studied. Recently, agronomic practices in development (early sowing, low tillage techniques, short rotations) are able to support the pathogen development. Today, a specific focus on agronomic aspects is strongly needed to try as much as possible to limit the use of the most risky. Genetics and plant breeding have been mainly used to increase winter oilseed rape resistance to blackleg. From 1977 to 1983, 0 + cultivars like Jet Neuf showed a high level of resistance. After the switch to 00 genotypes, the first released cultivars were more susceptible to blackleg disease. A useful step was reached, using a specific resistance gene (RIm1) for which the pathogen populations were mainly avirulent. Cultivars like Capitol, have been great commercial 
success all over Europe. After being widely used by plant breeders and cropped by farmers, this resistance became inefficient because of the increase of virulent races in pathogen populations. Resistant cultivars bearing RIm1 gene then appeared susceptible. Such a situation worried the different actors and raised the question of the development of adapted strategies for breeding and managing stable resistances to the disease in space and in time. Based on recent scientific works, we may distinguish two different types of resistance to Leptosphaeria maculans.

\section{Specific resistances}

Specific resistances are able to control ascospores penetration and leaf spot occurrence for L. maculans races having the avirulent allele (AvrLm) corresponding to the plant resistance gene $(\mathrm{R} / \mathrm{m})$. Such resistances involve gene-for-gene interactions between the pathogen and its host plant [1]. Such a specific plant resistance is very efficient when the fungus populations are mainly avirulent for this resistance. Specific resistances are quite easy to use for plant breeders because of their simple monogenic genetic determinism. When such a specific resistance is widely used, the development of the avirulent populations is reduced whereas the virulent populations are selected. After several years, the equilibrium between avirulent and virulent populations is moving towards the virulents. This is what probably happened in France with the specific resistance RIm1 in natural field conditions [2]. Experimental studies also demonstrated for other specific resistance gene (RIm6) that L. maculans population are able to adapt rather quickly, in 3 or 4 years time, to such specific resistances $[3,4]$. Constant efforts of research are done to find new efficient specific resistances, especially in neighbouring species like $B$. juncea or $B$. nigra $[5,6]$. Until now, nine different specific resistances are distinguished with corresponding reference cultivars. Three of them have no interest in France because the fungus populations are $100 \%$ virulent (RIm2, RIm3, RIm9). RIm1 and RIm4 have low and partial efficiency due to a majority of virulent isolates. Four known specific resistances (RIm5, RIm6, RIm7, RIm8) are both not used at a commercial level, and potentially efficient as the fungus populations are mainly avirulent. These genetic ressources have to be used carefully in the future for sustainable management. Two of them are coming from B. juncea. Probably new specific and potentially efficient resistances will be identified in the next future.

\section{Quantitative resistances}

Quantitative resistances are also generally involved in plant resistance to the pathogen. Its efficiency is partial, and its genetic determinism complex under control of several QTLs [7, 8]. Resistance mechanisms implied remain unknown. One of the most famous quantitative resistance sources to $L$. maculans in winter oilseed rape is Jet Neuf, a 0 + cultivar widely cropped during the seventies and the eighties all over Europe, still resistant to Leptosphaeria. When Jet Neuf is introduced in field trials today in France, it is still very resistant. With the first new 00 cultivars, this resistance was probably lost. Today, new varieties probably include levels of quantitative resistances which are always difficult to identify and to distinguish from specific resistances. Current work is carried out for genetic and molecular characterisation which will be helpful for plant breeders to select the QTLs implied in Darmor or Jet Neuf quantitative resistance. The advantage of such quantitative resistance is that they are probably much stable in time because they do not exert a selective pressure on the fungus populations. That is the reason why the promotion of such resistances is our priority. Nevertheless there is no proper way to estimate quantitative resistance levels in cultivars. Only an indirect rough estimation is possible through the confrontation of data (i) on general behaviour in the field, (ii) on 
owned specific resistances, which is possible to check with the use of the proper reference isolates in controlled conditions assays (iii) on leaf spot frequency in contaminated natural environment, and (iv) on virulence profiles of the fungus populations in the experimental sites.

\section{Pathogen populations}

Since the early nineties a huge effort has been done for a better characterisation of the pathogen population diversity. This type of study is mainly based on cotyledon inoculation tests on differential host genotypes, as first suggested by Williams [9]. In a first step a differential of 3 plant genotypes was used. New differential host genotypes were regularly added due to an increasing knowledge on the genetic bases of the interactions [10-12]. At present, 9 host genotypes are used to characterise the virulence profiles of Leptosphaeria isolates. Each genotype corresponds to only one, or a few known specific resistances. A national survey supported by the French Ministry of Agriculture is currently conducted to characterise populations in about 20 sites used for cultivar evaluations and for breeding. For each site, 100 isolates were isolated from leaf spots of a genotype known to have no specific or quantitative resistance to avoid genotypic effect on the sampling. Results show a large diversity among sites. The main question is how the pathogene population structure will now evolve under the genetic pressure provided by the cropped genotypes in the following years. Such a question can only be addressed by regular population surveys. Nevertheless this type of work is heavy and expensive and could not be applied to a larger scale. For the 3 main virulences of interest, PCR markers are under preparation and validation in order, to enable us to quickly type the fungus from leaf spots.

Such analyse, were also carried out for L. maculans isolate collections done by INRA and CETIOM during the nineties [2]. Interesting results were found for avrLm1 and avrLm4 virulences. Isolates were mainly AvrLm1 (avirulent) until 1997-98 and became quickly virulent (avrLm1) later, probably due to a wide use of genotypes carrying RIm1 specific resistance gene. On the other hand, RIm4 is a specific resistance which has been widely used in old varieties for many years, but not much during the nineties. L. maculans populations were mainly virulent until 1996-97, but a significant proportion of avirulent (AvrLm4) isolates were detected in the last years [2]. This is of great interest in the perspective of a sustainable management of genetic ressources and of the qualitative management of the pathogen populations. We may state the hypothesis that with a proper management of the genetic pressure, the development of the virulent population can be stopped, or at least reduced and perhaps the avirulent increased to sustain the partial efficiency of such specific resistance.

\section{Farmers level}

At the farmer level, varieties carrying RIm1 specific resistance were widely used with a great efficiency from 1995 to 1998 or 1999. During the two following years several genotypes known to be very resistant to $L$. maculans became rapidly susceptible. This was probably due to several reasons. Among them, agronomic practices have strongly moved to earlier sowings and low or no tillage techniques. Such an evolution has probably increased the contamination potential of many areas. The increase of the fungal inoculum may have contributed to the rapid specific resistance breakdown, especially on genotypes which did not have a sufficient quantitative resistance. This unpredicted change was very damaging to farmers but also for breeding, registration and extension 
bodies, and for their credibility towards farmers and end users. Nevertheless they have to continue their work by testing new genotypes and providing confident data and stable behaviour of genotypes towards L. maculans.

The situation at the farmer's level and the available knowledge suggest that it is necessary to try to promote a durable management of genetic resistances to Leptosphaeria, in close connection with strong messages on the agronomic techniques able to reduce the impact of the disease.

\section{Towards a durable management of genetic resistances}

Promoting a durable management of resistances is a complex objective which concerns the breeding of the varieties, their registration process, as well as the way to choose them, to sell or to use them, taking into account knowledge on resistance genetics and on pathogen population dynamics and interactions with the environment.

1) First we need a strategy to minimise the disease impact and make the resistance use as long and stable as possible. The first element of our strategy is clearly to promote as much as possible high levels of quantitative resistances which are able to reduce the breakdown risk. We do not know yet what could be the best general strategy, including or not specific resistances. This needs more knowledge on fungus epidemiology and population genetics, plant genetics, additive effects of specific and/or quantitative resistances. The European project SECURE will widely contribute to address these questions. Varietal associations of genotypes owning different resistances are also under study. Nevertheless, even if the best strategy is not already known, this has not to be a reason of immobilism. With a more detailed characterisation of genotypes it would be probably quite easy to avoid mistakes such as the one made collectively by ignorance in the case of RIm1. It would probably be beneficial to diversify cultivars in space and time and to avoid that only 2 or 3 genotypes cover the whole market. Alternance and diversity among cultivars would be the first message to address to end users. The definition of groups of genotypes developed on the knowledge available on their resistances could be very useful to promote alternance among genotypes different enough to maintain pathogen diversity and then efficiency.

2) Of course such a message requires that the varietal resistances are better known and identified in the varieties. It is possible and quite easy to test genotypes for specific resistances by using characterised isolates of the pathogen. Cotyledon tests with reference isolates allow to get the resistance profile for each tested variety. Such characterisation is in progress. We can quite easily imagine that such an information could be produced during the registration process of the new varieties, and then makes this knowledge available to the public. It is of public interest and of official duty to prevent the risks and give all available and useful information to users. As previously discussed, it is more difficult to characterise quantitative resistance which could be estimated only through indirect methods. QTLs molecular markers have been developed but they are not precise enough yet nor validated on a wide range of genotypes, to be used properly for genotype characterisation. It would be necessary to also try to develop controlled condition assays for this kind of resistance.

3) It is important to stress that agronomic pratices also have a tremendous role to play for limiting the blackleg stem canker risk and for improving the use of specific resistance gene. Stubble is the main medium on which the fungus survives and produces ascospores. Stubble management is a key 
question to try to control quantitative and qualitative evolutions of the pathogen populations. This is the reason why any agronomic practices able to modify the quantity, state or speed of degradation of stubbles are very important to be taken into account. Interactions between epidemic process and cultural practices remain partially unknown and are not enough quantified to predict the disease behaviour and the risks of resistance breakdown. At this level spatial approaches are also needed to take into account ascospores dissemination from field to field in a given area. Other aspects like sowing dates, plant density or nitrogen avaibility also have to be taken into account [13]. Research works are still going on in France and in Europe.

4) Local characterisation of the fungus populations on its virulence profiles could be very useful to adapt the varietal choice for a given area, especially if we want to consider partial effects of specific resistances when the corresponding avirulent population still represents a significant fraction of the total population. In addition, a site characterisation could be very interesting and useful for the main breeding and evaluation locations to get a better comprehension of genotype resistance, or to carry out a more secure genotype evaluation and have the proper result interpretation. However, as previously discussed, such a characterisation is time-consuming and expensive, even if molecular markers are available for race identification. Nevertheless, such characterisation has to be carried out periodically to follow the main populations with time.

5) The commercial actors, seeds companies, cooperatives, farmers are also eminent actors of a promotion of durable management of plant resistance to blackleg stem canker. In their negotiations to buy or sell a variety, the main element taken into account is the short- term profitability which contributes to reduce the number of chosen varieties in each area. In any case, the varietal diversification is not yet a factor of choice. The promotion of a durable management of resistances to Leptosphaeria also needs (i) to go deeper in to technical and scientific considerations in the commercial discussions and (ii) to take into account profitability on a long or midterm period. This needs modifications in the way people are discussing and are making decisions. Confidence and simplicity of the knowledge to be taken into account are important factors for commercial actors. Are they ready to integrate mid and longterm considerations ? Another difficulty is that topics in discussion among these actors are mainly around commercial products like varieties or fungicides. But adapted agronomic practices, on which no money would be owned, are not always a topic of first importance for economic. This will probably need a lot of communication and teaching skills on technical and scientific aspects from institutes like CETIOM.

\section{RÉFÉRENCES}

1. Manque. Ansan-Melayah, 1998.

2. Rouxel T, Penaud A, Pinochet X, Brun H, Gout L, Delourme R, Schmit J, Balesdent MH. A ten-year survey of populations of Leptosphaeria maculans in France indicates a rapid adaptation towards the RIm1 resistance gene of oilseed rape. Eur J Plant Pathol 2003 : (in press).

3. Brun $H$, Levivier $S$, Ruer $D$, Somda I, Chèvre AM, Renard M. A field method for evaluating the potential durability of new resistance sources : application to the Leptosphaeria maculans/Brassica napus pathosystem. Phytopathology $2000 ; 90$ : 961-6. 
4. Brun $H$, Pinochet $X$, Tanguy $X$, Caceres F, Sauzet $G$, Renard $M$. The potential durability of the resistance to Leptosphaeria maculans conferred to Brassica napus by a new major gene originating from B.juncea B genome depends on the Brassica napus genetic background. $13^{\text {th }}$ Crucifere Genetics Workshop, 23-26.03.2002, Davis, California, USA 2002 : 17.

5. Chèvre AM, Barret $P$, Eber F, Dupuy $P$, Brun $H$, Tanguy $X$, Renard M. Selection of stable Brassica napus-B. juncea recombinant lines resistant to blackleg (Leptosphaeria maculans) 1 : Identification of molecular markers, chromosomal and genomic origin of the introgression. Theor. Appl. Genet. 1997 ; 95 : 1104-11.

6. Chèvre AM, Eber F, This $P$, Barret $P$, Tanguy $X$, Brun $H$, Delseny $M$, Renard M. Characterization of Brassica nigra chromosomes and of blackleg resistance from $B$. nigra-B. napus addition lines. Plant Breeding $1996 ; 115: 113-8$.

7. Pilet ML, Delourme R, Foisset $N$, Renard M. Identification of loci contributing to quantitative field resistance to blackleg disease, causal agent Leptosphaeria maculans (Desm.) Ces. et de Not., in winter rapeseed (Brassica napus L.). Theor Appl Genet 1998 ; 96 : 23-30.

8. Pilet ML, Duplan G, Archipiano M, Barret P, Baron C, Horvais R, Tanguy X, Lucas MO, Renard M, Delourme R. Stability of QTL for field resistance to blackleg across two genetic backgrounds in rapeseed. Crop Sci $2001 ; 41: 197-205$.

9. Williams PH, Delwiche PA. Screening for resistance to blackleg of crucifers in the seedling stage. In Proc Eucarpia Conf Breed Cruciferous Crops, Wageningen, Pays Bas 1979 : 164-70.

10. Kuswinanti T, Sock J, Hoppe HH. Variation in virulence of aggressive isolates of Leptosphaeria maculans based on cotyledon reactions on an extended differential set. In : Proc $9^{\text {th }}$ Int Rapeseed Congress. 4-7 July 1995, Cambridge, GB 1995 : pp 1248-50.

11. Balesdent MH, Attard A, Ansan-Melayah D, Delourme R, Renard M, Rouxel T. Genetic control and host range of avirulence towards Brassica napus cvs. Quinta and Jet Neuf in Leptosphaeria maculans. Phytopathology $2001 ; 91: 70-6$.

12. Balesdent HM, Attard A, Khn ML, Rouxel T. New avirulence genes in the phytopathogenic fungus Leptosphaeria maculans. Phytopathology 2002 ; 92 (10) : 1122-33.

13. Aubertot JN, Crivineanu C, Le Floch D, Doré T. Analysis of the effects of the sowing date and nitrogen availability during autumn on blackleg development on two oilseed rape cultivars. $13^{\text {th }}$ Crucifer Genetics Workshop, March 23-26, Davis, USA 2002. 
A European research project - SECURE (Sept 2002 - Sept 2006)

"Stem canker of oilseed rape: molecular tools and mathematical modelling to deploy durable resistance"

Aims and objectives:

Stem canker of crucifers, also termed blackleg or phoma of oilseed rape, is caused by a complex of ascomycotous fungi collectively named Leptosphaeria maculans (anamorph: Phoma lingam) and is a disease of major economic importance. The Brassica napus-Leptosphaeria maculans pathosystem is governed by a gene-for-gene relationship. Major gene resistance has been very efficient in the past and this strategy has been highly profitable for both seed companies and the grower. However, with the narrow genetic basis associated with Brassica napus, resistance genes are rare and resistance under field conditions has been short lived. There is a need for resistance to be carefully managed to increase durability. SECURE (Stem canker of oilseed rape: molecular tools and mathematical modelling to deploy durable resistance) aims to deliver a model for the "managed" deployment of cultivars with resistance to Leptosphaeria maculans (phoma stem canker/blackleg) to improve durability of resistance and minimise risk that the resistance will break down.

\section{A wide Partnership:}

\section{United Kindom:}

+ Rothamsted Research, Harpenden, Herts., AL5 2JQ, U.K.:

\section{Coordinator}

Neal Evans, Bruce D.L. Fitt, Frank van den Bosch

+ ADAS Boxworth, Cambridge, CB3 8NN, U.K: Peter Gladders

France:

+ INRA, Unité PMDV, Route de St Cyr, 78026 Versailles, France :

Thierry Rouxel. Marie-Helene Balesdent

+ INRA UMR BiO3P, Domaine de la Motte, BP 35327, 35653 Le Rheu Cedex, France : Hortense Brun, Didier Andrivon

+ CETIOM, Centre de Grignon, BP no. 4, 78850 Thiverval-Grignon, France : Xavier Pinochet, Annette Penaud

Poland:

+ Instytut Genetyki Roslin, Strzeszynska 34, Poznan, 60-479, Poland Malgorzata Jedryczka, Piotr Kachlicki

\section{Sweden:}

+ Svalöf Weibull AB, Svalöv, SE-268 81, Sweden

Ingrid Happstadius, Jan Meyer 


\section{Five Work Packages:}

WP1: Modelling the life cycle of $L$. maculans. A mechanistic model ${ }^{1}$ of the life cycle of $L$. maculans will be produced. This needs to estimate values of model parameters (ascospore infection conditions, latent period to phoma leaf spot formation, growth of fungus down through petiole, canker establishment in stem and canker expansion), using existing data from experiments on five stages in the pathogen life cycle, in relation to temperature and wetness. This model will be tested qualitatively using existing data and new data from WP3.

WP2: Effects of pathogen variation at Avr loci on durability of resistance. Three avirulence genes, AvrLm6, AvrLm1 and AvrLm4, avirulence genes located in dissimilar genome environments, will be analysed. The analysis will include cloning and functional characterization of the gene and its allelic variants (AvrLm1 and AvrLm6) or sequencing of the genome region encompassing the gene (AvrLm4). An analysis of molecular events leading to virulence will be done. The fitness of the main allelic variants and near-isogenic lines of $L$. maculans will be assessed in controlled environment and glasshouse experiments.

WP3: Effects of genotype/environment on durability of resistance. Influence of plant genetic background and of the environment on durability of resistance will be analysed. First we shall survey distribution of $L$. maculans races in major oilseed rape producing regions of Europe, especially virulence against $R \operatorname{lm} 6$. Using near isogenic material with the resistance gene in different genetic backgrounds (i.e. EurolMX, DarmorMX) we shall perform recurrent selection (i.e. the same cultivar will be used each year to maximise selection pressure) on the pathogen populations to assess the potential durability (security, longevity, effectiveness) of R/m6 in different backgrounds at two sites (in west and east Europe). Durability (effectiveness $x$ duration $\mathrm{x}$ intensity of exposure) of the $R / m 6$ resistance in multi-site experiments at places where avirulent populations of $L$. maculans are predominant will be assess and we shall try to determine the "Iongevity" of this resistance. Evaluation of the effects of environmental factors (notably leaf temperature, wetness duration) on the expression of major gene/polygenic resistance in leaves/petioles of young plants will be done.

WP4: Strategy for sustainable deployment of durable resistance. We aim to model interactions between resistance and avirulent/virulent L.maculans races, to develop new criteria to assess durability of resistance (security, longevity, effectiveness), and to apply the models to predict effects of deployment strategy on durability of new resistance genes. This will allow to make recommendations for deployment of resistance to maximise durability of resistance genes.

WP5: Management and diffusion of results. New results and recommendations for deployment of resistance will be delivered to the end user by different means, including a regularly updated, interactive web-site (http://www.secure.rothamsted.ac.uk/), agricultural press articles, workshops, field demonstrations, new breeding strategies, conference presentations and refereed papers.For contact or more details please contact the coordinator Dr Neal Evans at the following address: neal.evans@bbsrc.ac.uk

${ }^{1}$ i.e. a model which seeks to represent the biological mechanisms underlying epidemic progress. 